\title{
石墨烯浓度对 $\mathrm{ZrH}_{1.8}$ 表面微弧氧化陶瓷层的影响
}

\author{
杜 培 ${ }^{1}$, 间淑芳 ${ }^{1,2}$, 陈伟东 ${ }^{1,2}$, 李世江 ${ }^{1}$, 马 文 ${ }^{1,2}$
}

(内蒙古工业大学 1. 材料科学与工程学院; 2. 内蒙古自治区薄膜与涂层重点实验室, 呼和浩特 010051)

摘 要: 在 $\mathrm{Na}_{5} \mathrm{P}_{3} \mathrm{O}_{10}-\mathrm{KOH}-\mathrm{Na}_{2} \mathrm{EDTA}$ 电解液中, 以石墨烯为添加剂, 在恒压模式下对 $\mathrm{ZrH}_{1.8}$ 表面进行微弧氧化处理。 采用涂层划痕仪测试陶瓷层与基体的结合力, 通过真空脱氢实验来评价陶瓷层的阻氢性能。电解液中添加石墨烯后, $\mathrm{ZrH}_{1.8}$ 表面微弧氧化陶瓷层均由内层致密层和外层疏松层构成, XRD 图谱显示, 所制陶瓷层主要由 $\mathrm{M}-\mathrm{ZrO}_{2}$ 和 $\mathrm{T}-\mathrm{ZrO}_{2}$ 相组成。随着石墨烯浓度的增加, 陶瓷层的氢渗透降低因子(Permeation Reduction Factor, PRF)呈先增大后减 小的趋势。当石墨烯浓度为 $0.10 \mathrm{~g} / \mathrm{L}$ 时, 陶瓷层的厚度约为 $66.5 \mu \mathrm{m}$, 表面孔洞和裂纹较少, 陶瓷层较致密, PRF 值 为 13.2 , 阻氢性能较好。

关 键 词: 氢化锆; 微弧氧化; 石墨烯; 陶瓷层; 阻氢性能

中图分类号: TG174 文献标识码: A

\section{Graphene Concentration on Micro-arc Oxidation Ceramic Layer of $\mathrm{ZrH}_{1.8}$ Surface}

\author{
DU Pei ${ }^{1}$, YAN Shu-Fang ${ }^{1,2}$, CHEN Wei-Dong ${ }^{1,2}$, LI Shi-Jiang ${ }^{1}$, MA Wen ${ }^{1,2}$
}

(1. College of Materials Science and Engineering, Inner Mongolia University of Technology, Hohhot 010051, China; 2. Inner Mongolia Key Laboratory of Thin Film and Coatings, Inner Mongolia University of Technology, Hohhot 010051, China)

\begin{abstract}
The surface of $\mathrm{ZrH}_{1.8}$ was subjected to micro-arc oxidation treatment in a constant pressure mode using graphene as an additive in $\mathrm{Na}_{5} \mathrm{P}_{3} \mathrm{O}_{10}-\mathrm{KOH}-\mathrm{Na}_{2}$ EDTA electrolyte. Adhesion of the ceramic layer to the substrate was tested using a coating scratch tester. Hydrogen barrier property of the ceramic layer were evaluated by vacuum dehydrogenation experiments. After adding graphene to the electrolyte, the micro-arc oxidation ceramic layer on the $\mathrm{ZrH}_{1.8}$ surface is composed of an inner dense layer and a loose outer layer. XRD pattern shows that the ceramic layer is mainly composed of $\mathrm{M}-\mathrm{ZrO}_{2}$ and $\mathrm{T}-\mathrm{ZrO}_{2}$ phases. As the concentration of graphene increases, the permeation reduction factor (PRF) of the ceramic layer increases firstly and then decreases. When the concentration of graphene is $0.10 \mathrm{~g} / \mathrm{L}$, the thickness of the ceramic layer is about $66.5 \mu \mathrm{m}$, with less surface pores and cracks, denser ceramic layer, PRF at 13.2, and better hydrogen barrier performance.
\end{abstract}

Key words: zirconium hydride; micro-arc oxidation; graphene; ceramic layer; hydrogen barrier performance

氢化锆具有低中子捕获截面、高中子散射截面、 高含氢量、良好的导热性和较强的抗辐射能力, 最 重要的是它具有负的温度系数, 即当反应堆温度升 高时, 其慢化效率下降, 从而使核裂变速度放慢, 能有效地控制反应堆的温度, 显著提高反应堆的安
全性 ${ }^{[1]}$ 。用氢化锆作慢化剂的反应堆能在较高温度 下工作而无需高压容器, 可以使反应堆的体积和质 量大大减小, 这对于制造空间和远程飞机等需要特 殊能源的微型反应堆具有重要意义 ${ }^{[2]}$ 。但氢化锆在 其工作温度(一般为 $650{ }^{\circ} \mathrm{C}$ 左右)下，反应平衡容易

收稿日期：2019-01-18; 收到修改稿日期：2019-05-14

基金项目：国家自然科学基金(51964035, 51364026) National Natural Science Foundation of China (51964035, 51364026)

作者简介：杜 培(1993-), 女, 硕士研究生. E-mail: 1991705267@qq.com

通讯作者：问淑芳，副教授. E-mail: ysfch@163.com 
向氢化锆分解的方向移动, 从而缩短了氢化锆作为 慢化剂的使用寿命。因此, 有必要在氢化锆表面制 备保护涂层阻挡或减缓氢的逸出, 以此来延长氢化 锆慢化剂的服役寿命 ${ }^{[3-4]}$ 。

目前, 氢化锆表面阻氢涂层的制备方法有电镀 法、溶胶一凝胶法、原位氧化和微弧氧化 ${ }^{[5-9]}$ 等。其 中, 微弧氧化法是在普通阳极氧化基础上, 利用弧 光放电增强并激活在阳极上发生的反应, 从而在 $\mathrm{Al} 、 \mathrm{Ti}$ 和 $\mathrm{Mg}$ 金属及其合金材料表面形成强化陶瓷 膜。这种方法工艺简单, 生产效率高, 可满足复杂零 件的制备, 形成的涂层与基体结合力较强, 而被广 泛应用 ${ }^{[10]}$ 。传统的微弧氧化技术制备的陶瓷层具有 疏松多孔结构，阻氢性能低。Yan 等 ${ }^{[11]}$ 在磷酸盐电 解液体系中, 采用恒压模式对氢化锆进行微弧氧化 处理, 可获得连续致密、表面缺陷较少的 $\mathrm{ZrO}_{2}$ 陶瓷 层; Chen 等 ${ }^{[12]}$ 在硅酸盐电解液中加入石墨烯纳米颗 粒, 降低了击穿电压, 加速电压的增长速率, 增加了 微弧氧化涂层的厚度, 使涂层的表面质量得到改善。

石墨烯是一种导电性能优异的二维材料, 电解 液中石墨烯的存在会增强微弧氧化过程电场强度, 从而加速石墨烯颗粒向涂层孔洞中沉积 ${ }^{[12-13]}$ 。微弧 氧化技术应用于氢化锆表面陶瓷层的制备已有报 道 ${ }^{[11,14]}$, 将石墨烯加入到电解液中, 研究其对氢化 锆表面微弧氧化陶瓷层影响的报道较少。本工作以 石墨烯为添加剂, 在磷酸盐体系中, 研究石墨烯浓 度对 $\mathrm{ZrH}_{1.8}$ 表面微弧氧化陶瓷层结构、形貌和阻氢 性能的影响。

\section{1 实验方法}

\section{1 试样准备}

用数控线切割机将圆柱形 $\mathrm{ZrH}_{1.8}$ 基体切割成尺 寸为 $\phi 20 \mathrm{~mm} \times 2 \mathrm{~mm}$ 的试样, 在距试样边缘 $2 \mathrm{~mm}$ 处 打一个 $\phi 3 \mathrm{~mm}$ 的孔, 然后将试样依次用 360 \#、600 \#、 800 \#、1000 \#、1200 \#的 $\mathrm{SiC}$ 水砂纸逐级打磨。同
时, 为了防止微弧氧化过程中出现尖端放电现象, 将试样边角打磨光滑。将打磨后的试样用无水乙醇、 丙酮和去离子水超声清洗各 $15 \mathrm{~min}$, 以除去表面的 油脂和杂质, 最后烘干备用。本实验中所有化学试 剂均为分析纯(AR)级, 所用的石墨烯由江南石墨烯 研究院提供。

石墨烯加入电解液之前, 用 $N, N$-二甲基甲酰胺 (DMF)作为分散剂超声分散 $5 \mathrm{~h}$, 再用磁力搅拌器摚 拌数小时。然后将分散较均匀的石墨烯分散液加入 到电解液中, 微弧氧化过程中一直用搅拌器对电解 液进行摚拌。

\section{2 电解液准备}

电解液采用磷酸盐体系, 以 $\mathrm{Na}_{5} \mathrm{P}_{3} \mathrm{O}_{10}$ 为主电解 质, $\mathrm{KOH} 、 \mathrm{Na}_{2}$ EDTA 等为辅助电解质, 选用去离子 水配制。微弧氧化过程中, 电解液温度通过冷却水 循环系统控制在 $25{ }^{\circ} \mathrm{C}$ 以下, 采用恒压模式, 电源正 负向电压、频率固定不变, 电流随反应过程自动调 节。电解液组成及微弧氧化实验参数如表 1 所示。

\section{3 微弧氧化陶瓷层的性能表征}

采用 HITACHI S-3400 扫描电子显微镜(SEM) 观察 $\mathrm{ZrH}_{1.8}$ 陶瓷层的表面及截面形貌; 通过 APD型全自动粉末 $\mathrm{X}$ 射线衍射仪 (XRD, 参数: $\mathrm{Cu}$ 靶, 电 流 $35 \mathrm{~mA}$, 电压 $40 \mathrm{kV}$, 步进扫描 $0.02^{\circ}$ ) 分析陶瓷层 相结构; 借助 ESCALAB 250Xi 型 X 射线光电子能 谱仪(XPS)分析陶瓷层的化学组成; 采用 WS-2005 涂 层附着力自动划痕仪测量陶瓷层与基体的结合力。

$\mathrm{ZrH}_{1.8}$ 表面陶瓷层的阻氢性能通过真空脱氢实 验进行表征, 将微弧氧化后的 $\mathrm{ZrH}_{1.8}$ 样品置于真空 度为 $1 \times 10^{-4} \mathrm{~Pa}$ 的真空环境中加热至 $650{ }^{\circ} \mathrm{C}$ 并保温 $50 \mathrm{~h}$, 然后通过测量试样的失氢量来评价 $\mathrm{ZrH}_{1.8}$ 表 面陶瓷层的阻氢性能, 用氢渗透降低因子(Permeation Reduction Factor, PRF) 表征 ${ }^{[11]}($ 式(1)):

$$
\mathrm{PRF}=Q_{0} / Q
$$

其中 $Q_{0}$ 为无陶瓷层时的失氢量, $Q$ 为有陶瓷层时的 失氢量。

表 1 电解液组成及微弧氧化实验参数

Table 1 Electrolyte composition and micro-arc oxidation experimental parameters

\begin{tabular}{|c|c|c|c|c|c|c|}
\hline $\begin{array}{c}\text { Graphene } \\
\text { concentration } /\left(g \cdot L^{-1}\right)\end{array}$ & Anode voltage/V & Cathode voltage/V & Frequency/Hz & Duty cycle & Time $/ \mathrm{min}$ & $\begin{array}{l}\text { Electrolyte } \\
\text { composition }\end{array}$ \\
\hline 0 & \multirow{5}{*}{430} & \multirow{5}{*}{150} & \multirow{5}{*}{150} & \multirow{5}{*}{50} & \multirow{5}{*}{15} & \multirow{5}{*}{$\begin{array}{c}\mathrm{Na}_{5} \mathrm{P}_{3} \mathrm{O}_{10} \\
\mathrm{KOH} \\
\mathrm{Na}_{2} \text { EDTA }\end{array}$} \\
\hline 0.05 & & & & & & \\
\hline 0.10 & & & & & & \\
\hline 0.15 & & & & & & \\
\hline 0.20 & & & & & & \\
\hline
\end{tabular}




\section{2 结果与讨论}

\section{1 石墨烯浓度对 $\mathrm{ZrH}_{1.8}$ 表面微弧氧化陶瓷 层表面形貌的影响}

通过扫描电子显微镜观察到的微弧氧化陶瓷层 的表面形貌如图 1 所示。从图 1(a)中可以看出, 在 未加入石墨烯时，微弧氧化制备的陶瓷层表面粗糙 且多孔，裂纹也较多。随着石墨烯浓度的增加，膜层 表面裂纹减少, 微孔数量增加, 如图 1(b)所示。继续 添加石墨烯，当浓度为 $0.10 \mathrm{~g} / \mathrm{L}$ 时，陶瓷层表面微 孔数量增加，孔径减小，孔口多呈闭合状，且裂纹 明显减少。这是由于随着放电击穿，吸附在 $\mathrm{ZrH}_{1.8}$ 表面的石墨烯被来自放电通道的熔融喷射物包裹住, 然后在电解液中淬火和固化; 还有一部分石墨烯沉 积到了陶瓷层的微孔和微裂纹中，从而减小了表面 微孔的尺寸。当石墨烯浓度为 $0.15 \mathrm{~g} / \mathrm{L}$ 时, 如图 1(d) 所示, 陶瓷层表面出现大的孔洞缺陷, 表面粗䊁度 增加。这是因为石墨烯具有较好的导电性, 可以有 效降低击穿电压，促进 “电子雪崩” 和微弧氧化反 应，产生的高能等离子体击穿已经形成的陶瓷层， 并产生更多的熔融氧化物, 这些熔融氧化物难以快 速填补 $\mathrm{ZrH}_{1.8}$ 基体表面的放电微孔，导致 $\mathrm{ZrH}_{1.8}$ 表 面孔洞尺寸增大。当石墨烯浓度为 $0.20 \mathrm{~g} / \mathrm{L}$ 时, 微 弧氧化反应进一步增强, 微孔被排出通道的大量熔 融喷射物堵塞，并有部分熔融物出现了 “过烧” 现 象，如图 1(e)所示。

\section{2 石墨烯浓度对 $\mathrm{ZrH}_{1.8}$ 表面微弧氧化陶瓷 层截面形貌和厚度的影响}

采用微弧氧化技术在 $\mathrm{ZrH}_{1.8}$ 表面制备陶瓷层的 截面形貌如图 2 所示。由图可知, $\mathrm{ZrH}_{1.8}$ 表面微弧氧 化陶瓷层由内层致密层和外层疏松层组成。当电解 液中未添加石墨烯时, 形成的陶瓷层如图 2(a)所示, 大尺寸孔洞较多, 微孔直径可达 $10 \mu \mathrm{m}$, 陶瓷层的 厚度为 $54.3 \mu \mathrm{m}$ 。随着石墨烯浓度的增加，陶瓷层的 厚度呈现先增大后减小的趋势。当石墨烯浓度为 $0.10 \mathrm{~g} / \mathrm{L}$ 时，陶瓷层的厚度为 $66.5 \mu \mathrm{m}$, 陶瓷层中微 孔和裂纹等缺陷相对较少。原因可能是石墨烯优异 的导电性促进了微弧氧化反应，此时形成了更多细 小的放电通道, 熔融氧化物较均匀地沉积到放电 通道中, 更好地填补了微弧氧化陶瓷层的微孔和 裂纹, 使得陶瓷层较为致密。从图 2(d)中可以看出, 虽然陶瓷层的厚度最大, 但陶瓷层整体较疏松, 微 孔和裂纹缺陷较多, 即使在致密层也存在较大的 孔洞，这是导致整个陶瓷层致密性下降的主要原因。

\section{3 石墨烯浓度对 $\mathrm{ZrH}_{1.8}$ 表面微弧氧化陶瓷 层物相组成的影响}

不同石墨烯浓度时 $\mathrm{ZrH}_{1.8}$ 表面微弧氧化陶瓷层 的相组成如图 3 所示。由图中可以看出, 所有陶瓷层 都由单斜相 $\mathrm{M}-\mathrm{ZrO}_{2}$ 和四方相 $\mathrm{T}-\mathrm{ZrO}_{2}$ 组成, 且以单 斜相 $\mathrm{M}-\mathrm{ZrO}_{2}$ 为主。石墨烯浓度的变化对陶瓷层的相 组成并无明显影响。 $\mathrm{M}-\mathrm{ZrO}_{2}$ 相为低温相, $\mathrm{T}-\mathrm{ZrO}_{2}$ 为 高温相，这两种氧化锆物相都具有致密的结构，对 阻氢性能有一定的提高。但是在 XRD 图谱中并没
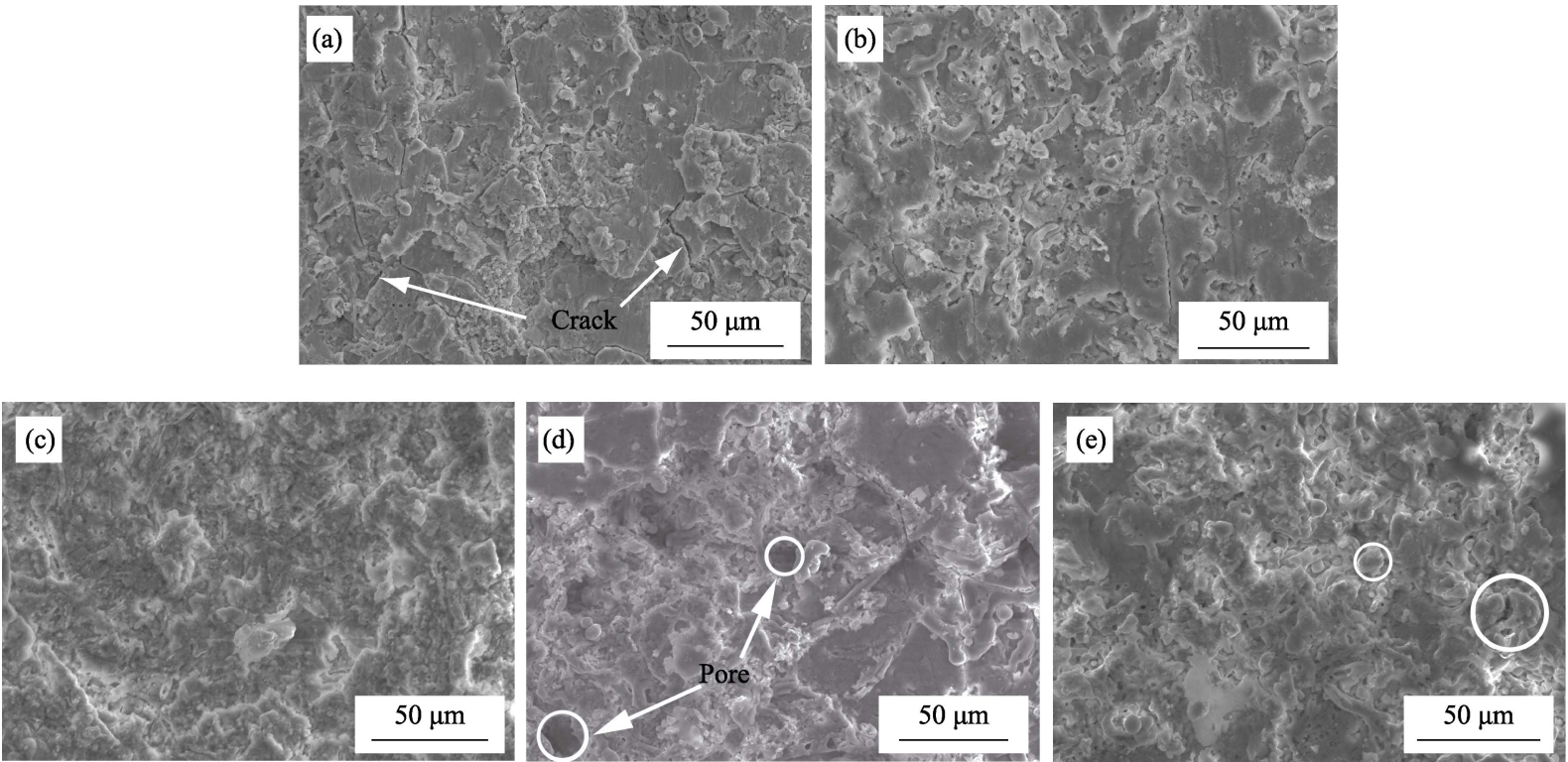

图 1 不同石墨烯浓度时微弧氧化陶瓷层的表面形貌

Fig. 1 Surface morphologies of micro-arc oxidation ceramic layer at different graphene concentrations (a) Without graphene; (b) $0.05 \mathrm{~g} / \mathrm{L}$; (c) $0.10 \mathrm{~g} / \mathrm{L}$; (d) $0.15 \mathrm{~g} / \mathrm{L}$; (e) $0.20 \mathrm{~g} / \mathrm{L}$ 

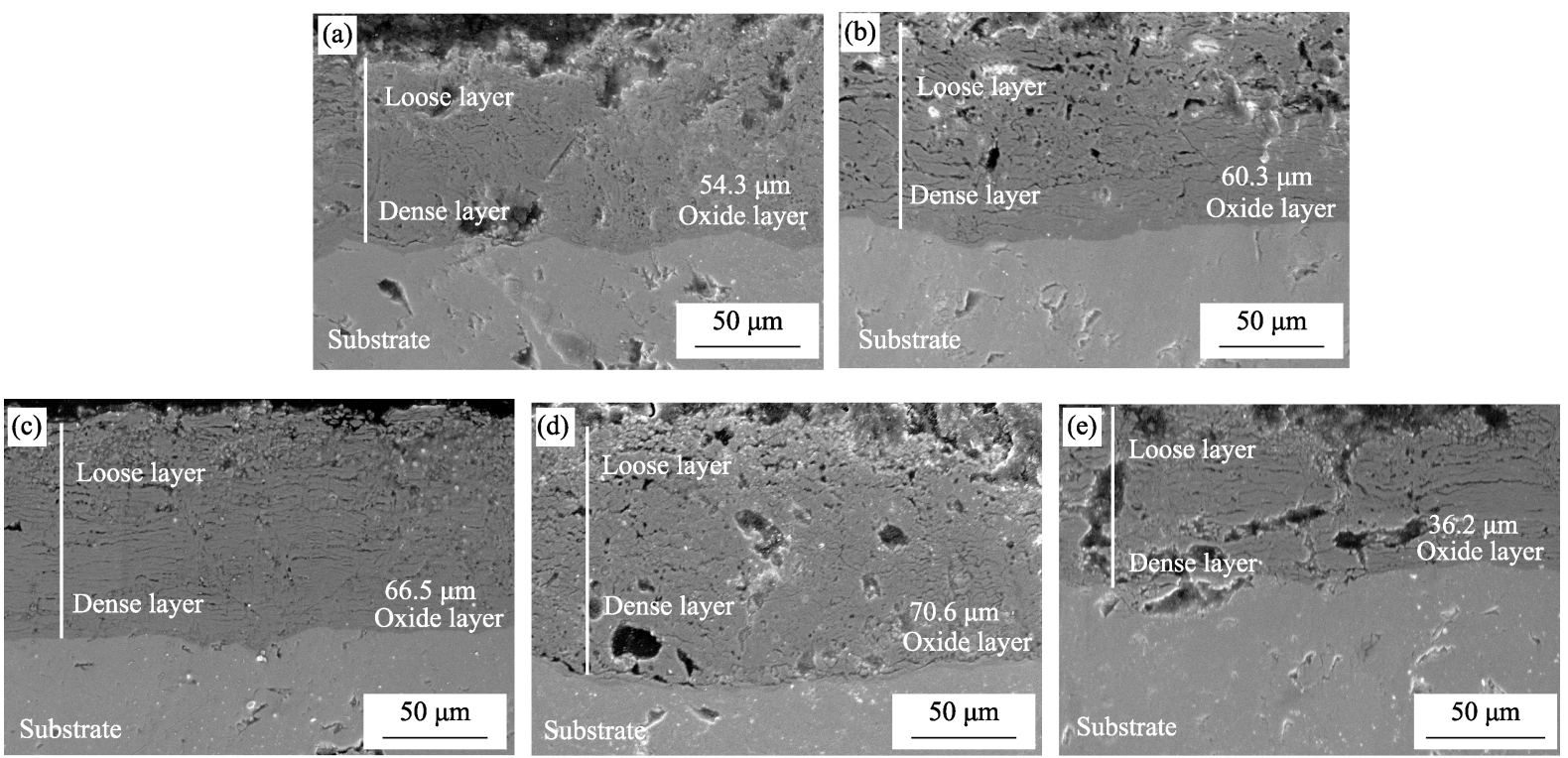

图 2 不同石墨烯浓度时微弧氧化陶瓷层的截面形貌

Fig. 2 Cross-section morphologies of micro-arc oxidation ceramic layer at different graphene concentrations (a) Without graphene; (b) $0.05 \mathrm{~g} / \mathrm{L}$; (c) $0.10 \mathrm{~g} / \mathrm{L}$; (d) $0.15 \mathrm{~g} / \mathrm{L}$; (e) $0.20 \mathrm{~g} / \mathrm{L}$

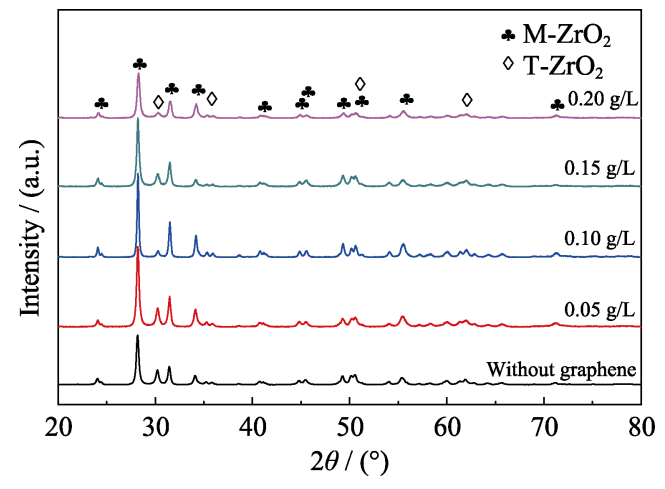

图 3 不同石墨烯浓度时微弧氧化陶瓷层的 XRD 图谱

Fig. 3 XRD patterns of micro-arc oxidation ceramic layer at different graphene concentrations

(a) Without graphene; (b) $0.05 \mathrm{~g} / \mathrm{L}$; (c) $0.10 \mathrm{~g} / \mathrm{L}$; (d) $0.15 \mathrm{~g} / \mathrm{L}$; (e) $0.20 \mathrm{~g} / \mathrm{L}$

有检测到石墨烯的衍射峰，这可能是因为微弧氧化 陶瓷层中石墨烯的含量太少所致，或者石墨烯衍射 峰受到氧化物的干扰。在本实验中, $\mathrm{M}-\mathrm{ZrO}_{2}$ 和 $\mathrm{T}-\mathrm{ZrO}_{2}$ 相的存在可能会影响石墨烯相的检测。

为了进一步确定微弧氧化陶瓷层中是否存在石 墨烯，对不同石墨烯浓度下制备的微弧氧化陶瓷层 进行 X 射线光电子能谱分析, 结果如图 4 所示。从 XPS 全谱扫描结果可以看出, 陶瓷层主要由 $\mathrm{C} 、 \mathrm{O}$ 、 $\mathrm{Zr}$ 等元素组成。图 4(a)中出现了结合能为 $284.95 \mathrm{eV}$ 的 C1s 信号, 这在环境中碳氢化合物的 XPS 表面扫 描中很常见。相比之下, 图 4(b)中 C1s 峰的强度显 著强于图 4(a)中 $\mathrm{C} 1 \mathrm{~s}$ 峰的强度, 这表明石墨烯进入 $\mathrm{ZrH}_{1.8}$ 表面微弧氧化陶瓷层中 ${ }^{[15-17]}$ 。

图 5 为图 4(b)陶瓷层中各元素的高分辨率光谱。 根据微弧氧化陶瓷层表面各元素的电子结合能可以
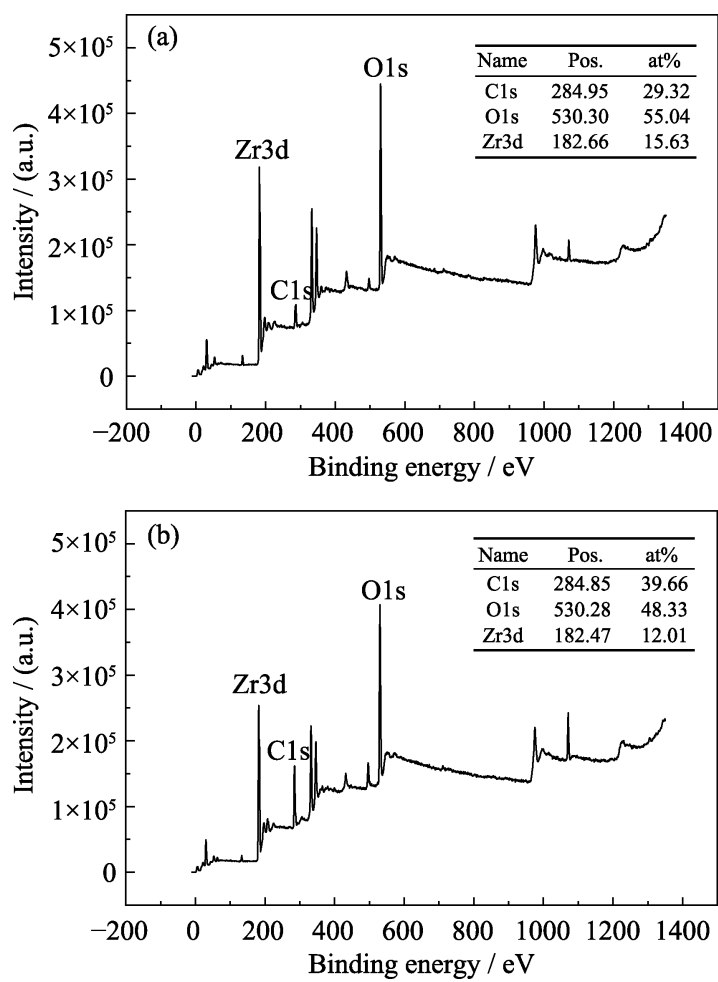

图 4 不同石墨烯浓度时微弧氧化陶瓷层的 XPS 图谱

Fig. 4 XPS spectra of micro-arc oxidation ceramic layer at different graphene concentrations

(a) Without graphene; (b) $0.10 \mathrm{~g} / \mathrm{L}$

推断氧化陶瓷层表面元素的存在形式。其中, 由图 5(a) 可知, $\mathrm{C} 1 \mathrm{~s}$ 的峰值为 $284.65 、 285.61 、 288.47 \mathrm{eV}$, 分 别对应于 $\mathrm{C}=\mathrm{C} 、 \mathrm{C} \mathrm{sp}{ }^{3} 、 \mathrm{O}-\mathrm{C}=\mathrm{O}$ 化学键，表明 $\mathrm{ZrH}_{1.8}$ 微弧氧化陶瓷层中存在石墨烯, 并且 $\mathrm{O}-\mathrm{C}=\mathrm{O}$ 化学 键的出现可能是由于在微弧氧化处理过程中石墨烯 的氧化; 在图 5(b)中 $\mathrm{O} 1 \mathrm{~s}$ 的峰值为 529.38 和 $531.08 \mathrm{eV}$, 
表明 $\mathrm{O}$ 元素以 $\mathrm{ZrO}_{2}$ 形式存在; 在图 5(c)中 $\mathrm{Zr} 3 \mathrm{~d}$ 的峰 值为 182 和 $183.3 \mathrm{eV}$, 表明 $\mathrm{Zr}$ 元素以 $\mathrm{ZrO}_{2}$ 形式存在。

\section{4 石墨烯浓度对 $\mathrm{ZrH}_{1.8}$ 表面微弧氧化陶瓷 层结合力的影响}

图 6 为电解液中不同石墨烯浓度下 $\mathrm{ZrH}_{1.8}$ 表面 微弧氧化陶瓷层与基体的结合力。由图中可以看出, 随着石墨烯浓度的增加, 陶瓷层与基体的结合力呈 现先增大后减小的趋势, 当石墨烯浓度为 $0.10 \mathrm{~g} / \mathrm{L}$ 时，陶瓷层与 $\mathrm{ZrH}_{1.8}$ 基体结合力达到最大，为 $65 \mathrm{~N}$ 。 这主要是由于石墨烯具有优异的导电性, 加入到电 解液中之后, 提高了溶液的的导电性, 加快了物质 的输送，使生成的陶瓷层与基体结合较为紧密，同 时微弧氧化过程中, 石墨烯沉积到陶瓷层的孔洞和 裂缝里, 使陶瓷层的致密性提高, 因而也提高了陶
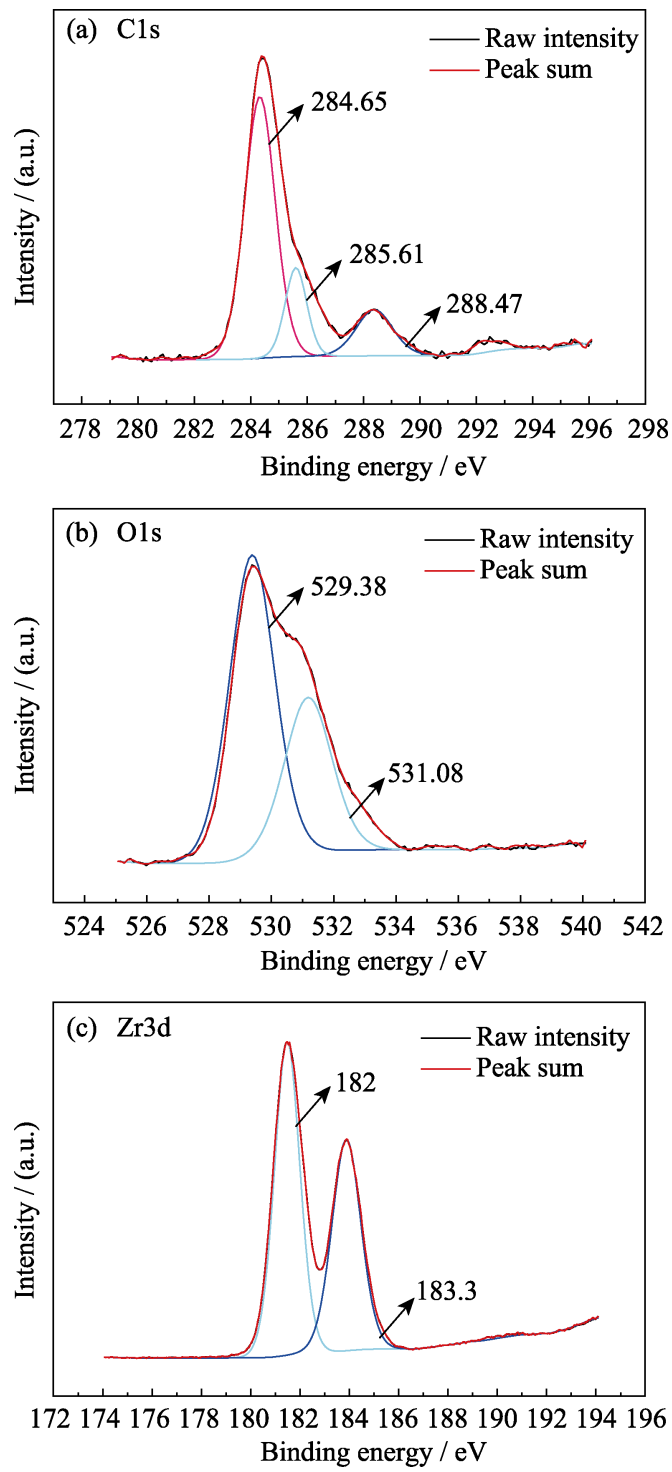

图 5 图 4(b)中检测到的元素的 XPS 高分辨率光谱

Fig. 5 XPS high resolution spectra of elements detected in Fig. 4(b) (a) $\mathrm{C}$; (b) $\mathrm{O}$; (c) $\mathrm{Zr}$

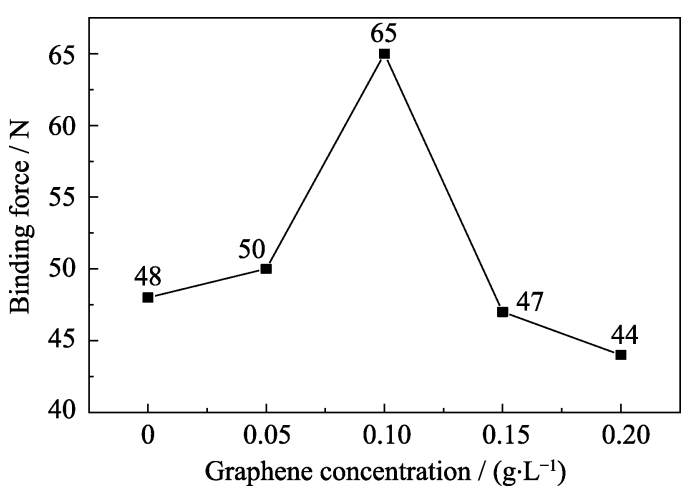

图 6 不同石墨烯浓度时微弧氧化陶瓷层与 $\mathrm{ZrH}_{1.8}$ 基体的结 合力

Fig. 6 Bonding force between micro-arc oxidation ceramic layer and $\mathrm{ZrH}_{1.8}$ matrix at different graphene concentrations

瓷层与基体的结合力。随着石墨烯浓度的继续增加, 电解液导电性过大，新生成的陶瓷层在强电压的作 用下再次击穿熔融，熔融氧化物来不及固化，使陶 瓷层的孔洞和裂纹增多，致密性下降，导致陶瓷层 与基体的结合力减小。

\section{5 石墨烯浓度对 $\mathrm{ZrH}_{1.8}$ 表面微弧氧化陶瓷 层阻氢性能的影响}

图 7 为电解液中不同石墨烯浓度下 $\mathrm{ZrH}_{1.8}$ 表面 微弧氧化陶瓷层的氢渗透降低因子 PRF，从图 7 中 可以看出, 电解液中未加入石墨烯时, 陶瓷层的 PRF 为 10.9 , 随着石墨烯浓度的增加, PRF 值先增大后减 小, 当石墨烯浓度是 $0.10 \mathrm{~g} / \mathrm{L}$ 时, PRF 值达到最大, 为 13.2 , 表明微弧氧化制得的陶瓷层的阻氢性能较 好。结合陶瓷层的表面形貌和截面形貌可知，此时 所制陶瓷层表面孔洞和裂纹缺陷少，孔径也较小， 陶瓷层较为致密, 使得氢渗透率较低。继续增加石 墨烯的浓度到 $0.20 \mathrm{~g} / \mathrm{L}$ 时, PRF 值减小为 8.5 , 阻氢 性能明显下降, 这主要是由于陶瓷层中存在较多的 孔洞和裂纹，导致陶瓷层的致密性显著降低。

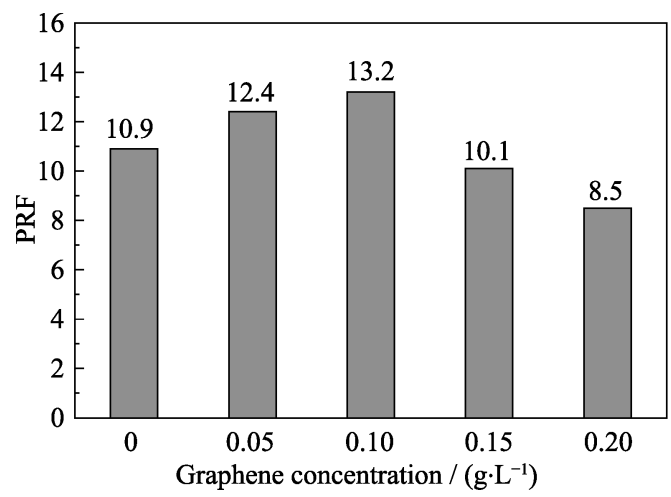

图 7 不同石墨烯浓度时微弧氧化陶瓷层的 PRF 值

Fig. 7 PRF value of micro-arc oxidation ceramic layer at different graphene concentrations 


\section{3 结论}

1) 恒压模式下，电解液中加入不同浓度石墨 烯在 $\mathrm{ZrH}_{1.8}$ 表面可制得厚度适中且较为致密的氧化 锆陶瓷层。

2) 不同石墨烯浓度下所制的微弧氧化陶瓷层 主要由 $\mathrm{M}-\mathrm{ZrO}_{2}$ 和 $\mathrm{T}-\mathrm{ZrO}_{2}$ 组成, 且 XPS 光谱证实了 在陶瓷层中存在石墨烯。

3) $\mathrm{ZrH}_{1.8}$ 表面微弧氧化陶瓷层由致密层和疏松 层组成。当石墨烯浓度为 $0.10 \mathrm{~g} / \mathrm{L}$ 时, 陶瓷层的阻 氢性能较好, PRF 值为 13.2 。

\section{参考文献:}

[1] HAYASHI T, TOBITA K, NAKAMORI Y, et al. Advanced neutron shielding material using zirconium borohydride and zirconium hydride. Journal of Nuclear Materials, 2009, 386-388: 119-121.

[2] MATHEUS A T, CHINTHAKA M S, PHILIP D E. Site specific dependencies of hydrogen concentrations in zirconium hydrides. Scripta Materialia, 2019, 158: 136-140.

[3] OLANDER D, GREENSPAN E, GARKISCH H D, et al. Uraniumzirconium hydride fuel properties. Nuclear Engineering and Design, 2009, 239(8): 1406-1424.

[4] KUMAR N A P K, SZPUNAR J A, HE Z. Preferential precipitation of hydrides in textured zircaloy-4 sheets. Journal of Nuclear Materials, 2010, 403(1/2/3): 101-107.

[5] 单丽梅. 氢化锆表面电镀铬膜阻止氢渗透的研究. 成都: 西华 大学硕士学位论文, 2007.

[6] WU M, PENG J Q, YAN G Q, et al. Preparation and properties of composite hydrogen permeation barrier on $\mathrm{ZrH}_{1.8}$ by Sol-Gel technique. Surface and Coatings Technology, 2018, 352: 159-165.

[7] PENG J Q, CHEN Y, WU M, et al. Hydrogen desorption behavior of the hydrides of $\mathrm{Zr}-\mathrm{Y}$ alloys under $\mathrm{Ar}$ and $\mathrm{CO}_{2}$ atmosphere. Journal of Alloys and Compounds, 2017, 693: 103-109.

[8] SANTOSH P S, ETSUSHI T, YOSHITAKA A, et al. Cathodic pulse breakdown of anodic films on aluminium in alkaline silicate electrolyte-understanding the role of cathodic half-cycle in AC plasma electrolytic oxidation. Corrosion Science, 2012, 55: 90-96.

[9] YANG W, XU D P, YAO X F, et al. Stable preparation and characterization of yellow micro arc oxidation coating on magnesium alloy. Journal of Alloys and Compounds, 2018, 745: 609-616.

[10] LI H, SUN Y Z, ZHANG J. Effect of $\mathrm{ZrO}_{2}$ particle on the performance of micro-arc oxidation coatings on $\mathrm{Ti}_{6} \mathrm{Al}_{4} \mathrm{~V}$. Applied Surface Science, 2015, 342: 183-190.

[11] YAN G Q, CHEN W D, ZHONG X K, et al. Properties of oxide coating on the surface of $\mathrm{ZrH}_{1.8}$ prepared by microarc oxidation with different positive voltages. Rare Metals, 2013, 32(2): 169-173.

[12] CHEN Q Z, JIANG Z Q, TANG S G, et al. Influence of graphene particles on the micro-arc oxidation behaviors of 6063 aluminum alloy and the coating properties. Applied Surface Science, 2017, 423: 939-950.

[13] CHEN F, ZHANG Y L, ZHANG Y, et al. Effect of graphene on micro-structure and properties of MAO coating prepared on $\mathrm{Mg}-\mathrm{Li}$ alloy. International Journal of Electrochemical Science, 2017, 12: 6081-6091.

[14] WANG Z G, CHEN W D, YAN S F, et al. Characterization of $\mathrm{ZrO}_{2}$ ceramic coatings on $\mathrm{ZrH}_{1.8}$ prepared in different electrolytes by micro-arc oxidation. Rare Metals, 2015, DOI: 10.1007/s12598015-0503-8

[15] CHANG L. Growth regularity of ceramic coating on magnesium alloy by plasma electrolytic oxidation. Journal of Alloys and Compounds, 2009, 468: 462-465.

[16] LÜ G H, CHEN H, GU W C, et al. Effects of graphite additives in electrolytes on the microstructure and corrosion resistance of alumina PEO coatings. Current Applied Physics, 2009, 9(2): 324-328.

[17] HAN B J, YANG Y, HUANG Z G, et al. A composite anodic coating containing graphene on AZ31 magnesium alloy. International Journal of Electrochemical Science, 2017, 12: 9829-9843. 